\title{
Confidence in Recognition Memory for Words: Dissociating Right Prefrontal Roles in Episodic Retrieval
}

\author{
R. N. A. Henson, M. D. Rugg, and T. Shallice \\ University College London
}

\section{R. J. Dolan}

University College London and Royal Free Hospital School of Medicine, London

\begin{abstract}
We used event-related functional magnetic resonance imaging (efMRI) to investigate brain regions showing differential responses as a function of confidence in an episodic word recognition task. Twelve healthy volunteers indicated whether their old-new judgments were made with high or low confidence. Hemodynamic responses associated with each judgment were modeled with an "early" and a "late" response function. As predicted by the monitoring hypothesis generated from a previous recognition study [Henson, R. N. A., Rugg, M. D., Shallice, T., Josephs, O., \& Dolan, R. J. (1999a). Recollection and familiarity in recognition memory: An event-related fMRI study. Journal of Neuroscience, 19, 3962-3972], a right dorsolateral prefrontal region showed a greater response to correct low- versus
\end{abstract}

\section{INTRODUCTION}

Numerous imaging studies have demonstrated activation of the right prefrontal cortex during episodic memory retrieval (for reviews see Buckner \& Koutstaal, 1998; Fletcher, Frith, \& Rugg, 1997), and several different hypotheses have been advanced concerning the functional role of these activations. These hypotheses include the adoption of a retrieval mode (Kapur, Craik, Brown, Houle, \& Tulving, 1995), expenditure of retrieval effort (Schacter, Alpert, Savage, Rauch, \& Albert, 1996), probability of successful retrieval (Rugg, Fletcher, Frith, Frackowiak, \& Dolan, 1996), and monitoring the products of retrieval cueing (Shallice et al., 1994). In many situations, however, particularly in the blocked designs adopted by the majority of these studies, the predictions of several of these hypotheses are confounded. In blocked studies that have manipulated the ratio of old to new words for example (e.g., Rugg et al., 1996; Nyberg et al., 1995), the degree of retrieval success and amount of retrieval monitoring are likely to be highly correlated. The advent of event-related functional correct high-confidence judgements. Several regions, including the precuneus, posterior cingulate, and left lateral parietal cortex, showed greater responses to correct old than correct new judgements. The anterior left and right prefrontal regions also showed an old-new difference, but for these regions the difference emerged relatively later in time. These results further support the proposal that different subregions of the prefrontal cortex subserve different functions during episodic retrieval. These functions are discussed in relation to a monitoring process, which operates when familiarity levels are close to response criterion and is associated with nonconfident judgements, and a recollective process, which is associated with the confident recognition of old words. magnetic resonance imaging (efMRI; Dale \& Buckner, 1997; Josephs, Turner, \& Friston, 1997; Zarahn, Aguirre, \& D'Esposito, 1997), which permits responses to be conditionalized on both the subjective and objective properties of individual trials, offers some resolution to this problem. In the present study, we used efMRI in an attempt to tease apart the neural correlates of retrieval success and retrieval monitoring by asking participants to indicate the confidence of each of their old-new judgements during a word recognition task. We propose that increases in regional activity associated with correct old versus correct new judgements reflect the consequences of retrieval success, whereas increases associated with correct low- versus correct high-confidence judgements reflect the consequences of retrieval monitoring.

This proposal is based on the findings of a previous efMRI study (Henson, Rugg, Shallice, Josephs, \& Dolan, 1999a), in which we attempted to identify regions associated with the recollective experience during recognition memory for words. Using the $\mathrm{R} /$ 
K procedure of Tulving (1985), we observed greater responses associated with $\mathrm{R}$ than $\mathrm{K}$ judgements in several cortical regions, including the anterior prefrontal cortex, lateral parietal cortex, and posterior cingulate. These regions were confined to the left hemisphere, however. The right dorsolateral prefrontal cortex showed the opposite pattern of greater responses to $\mathrm{K}$ than $\mathrm{R}$ judgements. We hypothesized that this enhanced right dorsolateral prefrontal activity reflected increased monitoring requirements when recognition decisions were uncertain (as is likely, for example, when judgements are based on a feeling of familiarity in the absence of recollection). This hypothesis is an extension of the concept of retrieval monitoring developed by Koriat and Goldsmith (1996) and Norman and Bobrow (1979), and incorporated into the retrieval model of Burgess and Shallice (1996) as part of the "memory editor" process. In the context of a yes/no recognition task, monitoring would include the examination of the products of memory retrieval (e.g., "is the retrieved information relevant?") and decision processes pertinent to the final judgment (e.g., "is the information sufficient for me to respond yes?").

In terms of signal detection models of recognition (e.g., Green \& Swets, 1966; Murdock, 1965), decisions are uncertain when memory strength is close to the response criterion. These models represent old and new items as overlapping distributions of "memory strength", upon which participants impose a criterion for making "old" versus "new" judgements (Figure 1). Judgements to items with strengths far from the response criterion (old or new) are made with high confidence, whereas judgements to items close to the response criterion are made with low confidence. Because memory strength is a continuum, these models illustrate that retrieval monitoring is not necessarily contingent on retrieval "success", as operationally defined by a correct "old" judgement. Rather, memory strength can be regarded as evidential, comprising many types of information retrieved in response to a recognition cue, which require monitoring for their validity and appropriateness.

Other models assume that more than one process underlies recognition memory (e.g., Jacoby, 1996; Mandler, 1980). The dual-process model of Atkinson and Juola (1974), for example, assumes that items with moderate levels of memory strength are subjected to a further set of retrieval operations. The dual-process model of Yonelinas, Dobbins, Szymanski, Dhaliwal and King (1996), on the other hand, assumes independent contributions of recollection and familiarity. Recollection is formalized as an all-or-none, threshold phenomenon (which would be associated with high-confidence old judgments). Familiarity, however, is formalized as a continuous quantity, equivalent to memory strength in signal detection models. Thus,

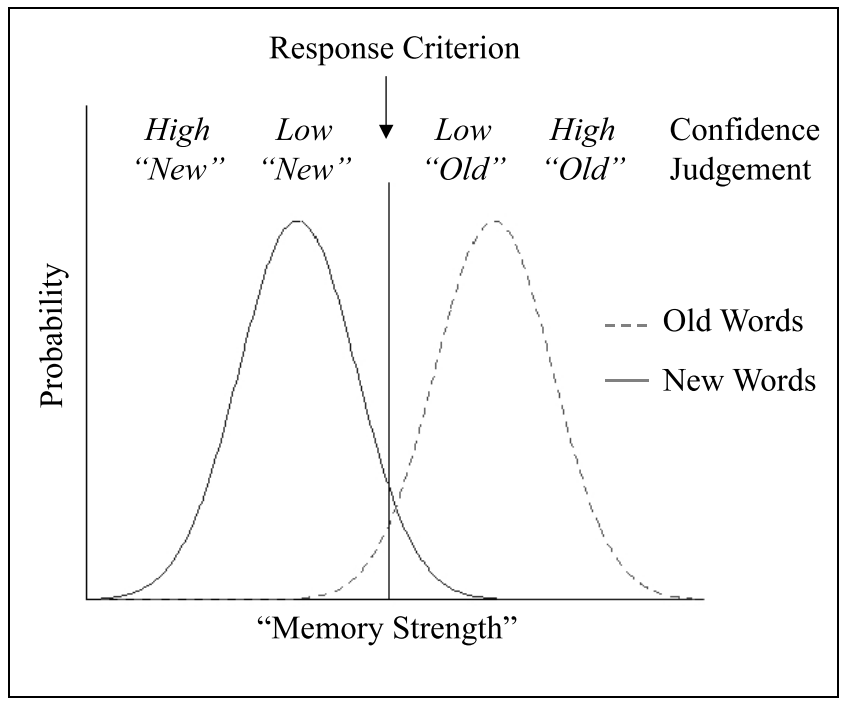

Figure 1. Schematic depiction of signal detection models of recognition judgements.

for both single and dual process models we would still expect greater monitoring when memory strengths (or familiarity levels) are close to the old-new response criterion.

We predicted therefore that the right dorsolateral prefrontal cortex (BA 9/46) would show greater responses associated with low- than high-confidence judgements, regardless of whether the word was old or new. We were also interested in the response characteristics of other right prefrontal regions implicated in previous studies, such as the anterior (BA 10) and ventral (BA 45/47) prefrontal cortex, which might show different response patterns, on the hypothesis that the right prefrontal cortex comprises several functionally dissociable subregions (Henson, Shallice, \& Dolan, 1999b).

\section{RESULTS}

\section{Behavioral Data}

The mean proportions and reaction times for correct judgements to old and new words are shown in Table 1. Collapsing across confidence, overall memory performance was good, as indexed by a Hit Rate-False Alarm rate of $0.91-0.17=0.74$. The proportions of correct new judgements made with low and high confidence were approximately equal, whereas a greater proportion of correct old judgements were made with high than low confidence, as confirmed by a significant interaction in a two-way ANOVA, $F(1,11)$ $=31.17, p<.001$. A two-way ANOVA on median correct reaction times showed significantly longer RTs for low- versus high-confidence judgements, $F(1,11)=$ $10.3, p<.01$, and for new versus old judgements, $F(1,11)=9.42, p<.05$, though the old-new difference was greater for confident old judgements, as 
Table 1. Proportions of Old and New Words and Reaction Times (RT) for Correct High- and Low-Confidence Judgements

\begin{tabular}{c} 
Word type \\
\hline Old \\
$\begin{array}{c}\text { High Low } \\
\text { Confidence Confidence }\end{array}$
\end{tabular}

Proportion

$\begin{array}{lrrrr}\text { Mean } & .73 & .18 & .42 & .41 \\ S D & .17 & .13 & .15 & .10\end{array}$

RT (msec)

\begin{tabular}{lllll} 
Mean & 1350 & 1939 & 1754 & 2032 \\
$S D$ & 223 & 613 & 470 & 657 \\
\hline
\end{tabular}

confirmed by the significant interaction, $F(1,11)=$ $6.95, p<.05$.

\section{Imaging Data}

Analyses were restricted to correct recognition judgments. Unfortunately, the relatively small numbers of correct low-confidence old judgements, together with their considerable variability across participants, precluded a full factorial analysis of the four categories of confident old, unconfident old, confident new, and unconfident new judgements (see Methods). To test the main effects of confidence or retrieval success, therefore, judgements were collapsed across old and new words, or across high- and low-confidence judgements, respectively.

The data were analyzed using two temporally shifted hemodynamic response functions (see Methods). This use of both "early" and "late" response functions was based on the concern that some right prefrontal re- sponses might be delayed and/or prolonged in time, as suggested both hemodynamically (Schacter, Buckner, Koutstaal, Dale, \& Rosen, 1997) and electrophysiologically (Wilding \& Rugg, 1996).

\section{Low Versus High Confidence}

A one-tailed planned comparison tested for regions showing a greater response to correct low- than correct high-confidence judgements (collapsing across old and new words). Given our prior monitoring hypothesis (Henson et al., 1999a), the results of the $\mathrm{K}$ versus $\mathrm{R}$ judgements in our previous study were used as a mask to define regions of interest for the present study (see Methods).

This comparison revealed a right dorsolateral prefrontal region (inferior frontal sulcus, BA 9/46, $x=54, y=$ $30, z=24$ ), which showed an enhanced early response for low-confidence judgements $(Z=2.69)$. This region survived $p<.05$ corrected for the shape and extent of the right dorsolateral region in the mask (25 voxels with an estimated isotropic spatial smoothness of $11 \mathrm{~mm}$ ). This region showed a slightly (but not significantly) prolonged differential response (Figure 2), as it also tended to load on the late $\operatorname{HRF}(Z=1.12)$. Post hoc tests of the simple effect on new words alone (for which there were similar numbers of correct high- and lowconfidence judgments) revealed that this region still showed a tendency for enhanced responses to lowconfidence judgements $(Z=1.41, p<.10$ and $Z=$ $1.84, p<.05$, for early and late HRFs, respectively).

A homologous dorsolateral prefrontal region in the left hemisphere (inferior frontal sulcus, BA 9/46, $x=$ $-39, y=21, z=24$ ) also showed an enhanced response to low-confidence judgments, collapsed across old and new words $(Z=3.24$ and $Z=1.64$ for early and late HRFs, respectively), though this
Figure 2. Right dorsolateral prefrontal region showing differential early responses to correct low- and correct highconfidence judgements (collapsed across old and new words, thresholded at $p<.01$, and masked with $\mathrm{K}-\mathrm{R}$ from Henson et al., 1999a, $p<.01$ ). The transverse slice is from a normalized T1 structural image of one participant's brain. The bar graph shows the mean and standard error of differences in low confidence versus high confidence parameter estimates for the early and orthogonalized late covariates from the maximum of the region. The event-related plot shows the resulting mean best-fitting, differential response as a function of peristimulus time (PST).

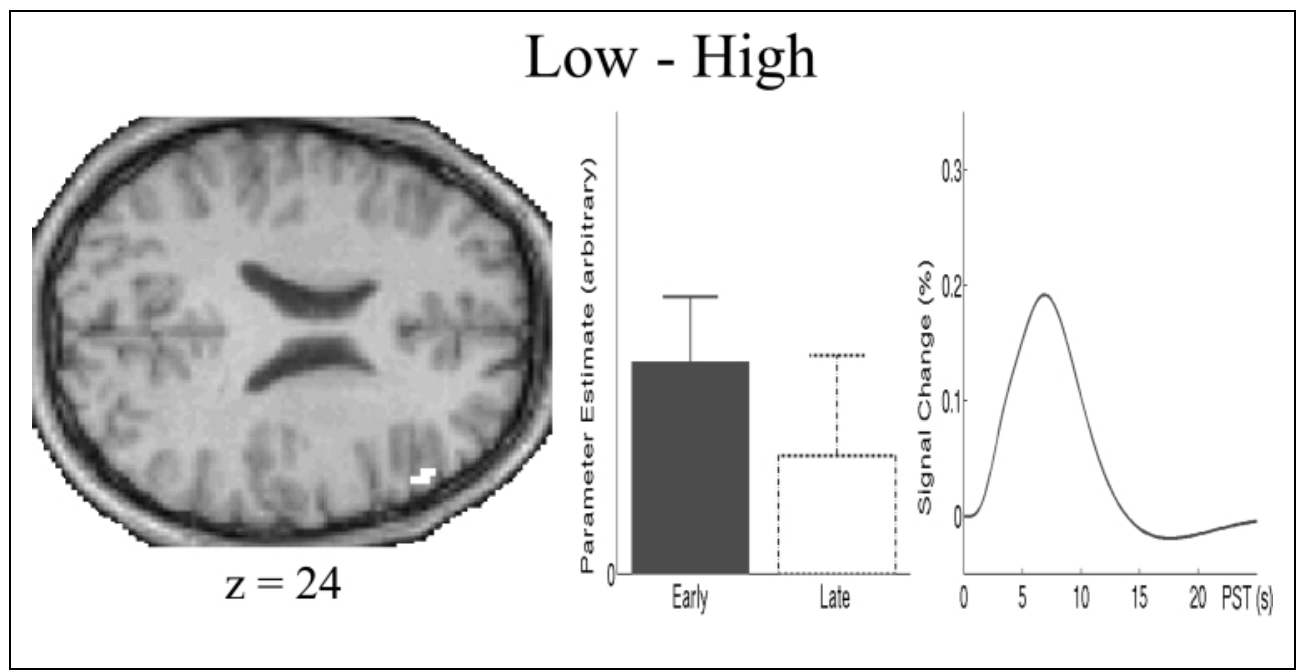

Henson et al. 
region was not identified in the $\mathrm{K}$ versus $\mathrm{R}$ comparison of our previous study.

\section{Old Versus New}

A one-tailed planned comparison tested for regions showing greater responses to correct old than correct new judgements (collapsed across high and low confidence). Regions of interest were defined by masking with the results of the corresponding comparison of old ( $\mathrm{R}$ and $\mathrm{K}$ ) versus new $(\mathrm{N})$ judgements in our previous study (Henson et al., 1999a; this specific comparison was not reported in that paper; see Methods).

Three posterior regions showed enhanced early responses to correct old judgements that survived $p<.05$ corrected for the extent of the corresponding regions in the mask. These were the left precuneus (BA 7, $x=0, y$ $=-69, z=33, Z=4.20$; mask region of 61 voxels), posterior cingulate (BA 23/30, $x=3, y=-42, z=21, Z$ $=3.95$; mask region of 15 voxels), and left lateral parietal cortex (inferior parietal gyrus, BA $40, x=-48, y=-57$, $z=48, Z=3.45$; mask region of 255 voxels). The precuneus (Figure $3 \mathrm{~A}$ ) and posterior cingulate regions
Figure 3. Regions showing differential early (A and B) or late $(\mathrm{C})$ responses to correct old and correct new judgements (collapsed across confidence, thresholded at $p<.01$, and masked with $\mathrm{R} / \mathrm{K}$ versus $\mathrm{N}$ from Henson et al., 1999a, $p<.01$ ). The bar graphs and event-related plots derive from maxima of the precuneus (A), left lateral parietal (B), and left anterior prefrontal (C) regions. For further details, see Figure 2 legend.

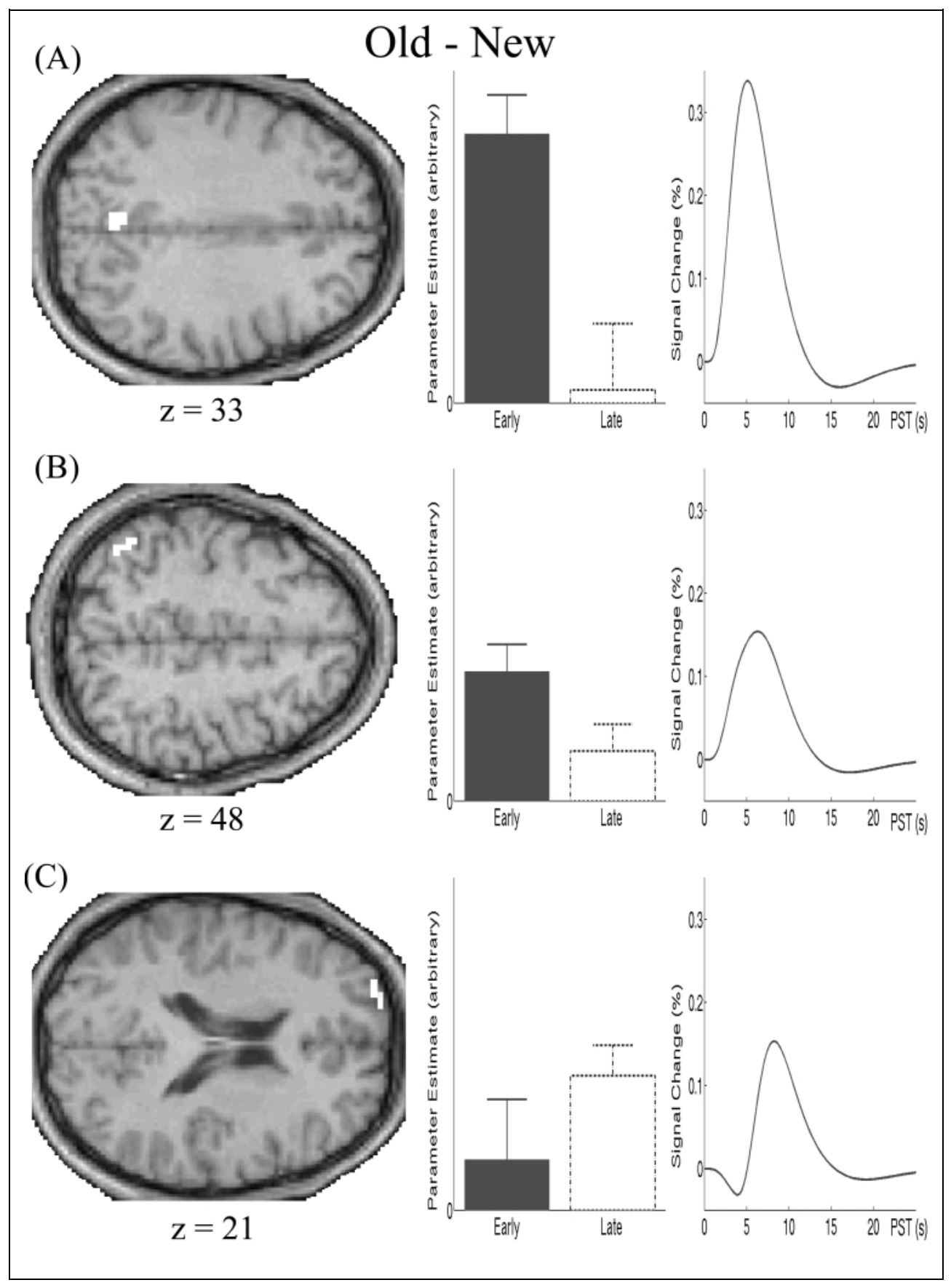


Figure 4. Anterior right prefrontal region (circled) showing differential late responses to correct old and correct new judgements (collapsed across confidence, thresholded at $p<$ $.01)$. The bar graphs and eventrelated plot derive from the maxima of the region. For further details, see Figure 2 legend.

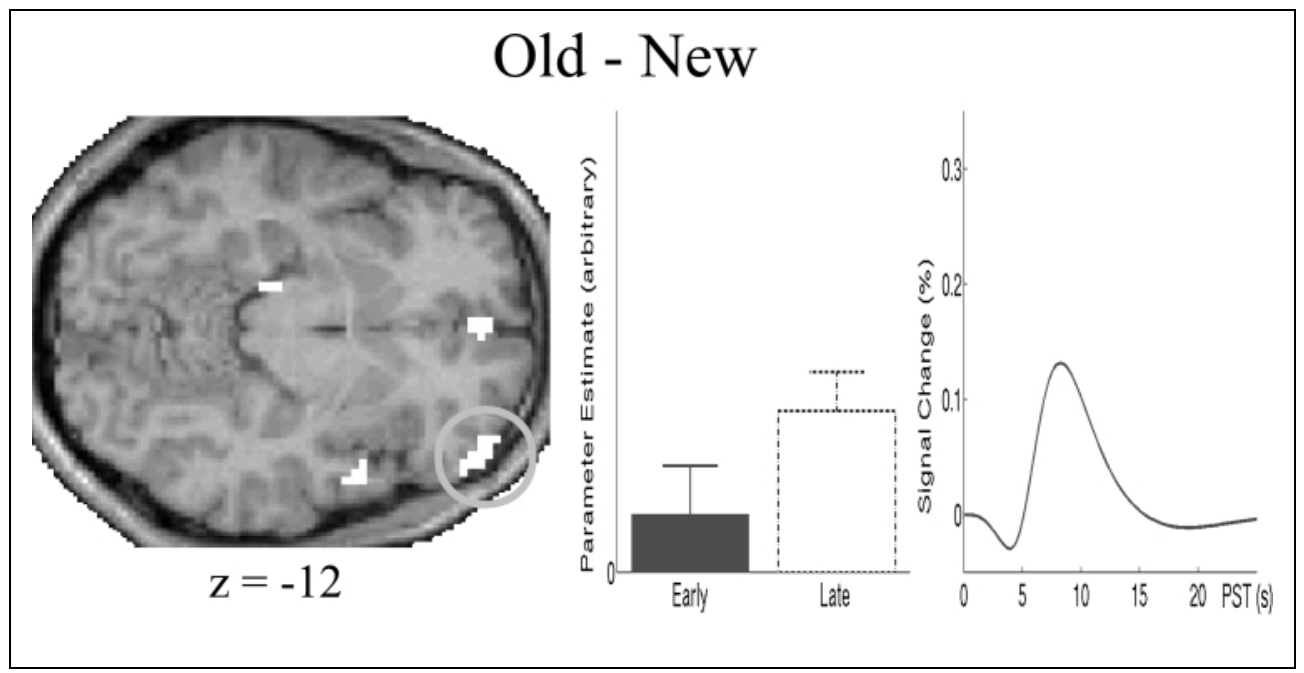

showed an early response, whereas the left lateral parietal region (Figure 3B) showed a more prolonged response, which also loaded on the late $\operatorname{HRF}(Z=1.71$, $p<.05)$.

The only region to survive small volume correction with the late HRF was the anterior left prefrontal cortex (superior frontal gyrus, BA 10, $x=-21, y=63, z=21$, $Z=3.28$; mask region of 39 voxels). This region showed a differential response that emerged relatively late in time (Figure 3C). Finally, a region in the right ventral anterior prefrontal cortex (anterior to clear definition of the superior frontal sulcus, BA 10/47, $x=48, y=48, z=$ $-12, Z=3.17$ ) also showed a late old-new effect (Figure $4)$, though this region did not survive masking. The delayed nature of this differential response may explain why this region was not identified in the corresponding comparison of our previous study (which used only an early HRF). While we regard this result as preliminary, we note that the nearby anterior right prefrontal regions (though generally more medial and superior) have been consistently activated during episodic retrieval (see Buckner \& Koutstaal, 1998) and have previously been associated with retrieval success in recognition memory tasks (Rugg et al., 1996; Rugg, Fletcher, Frith, Frackowiak, \& Dolan, 1997; Rugg, Fletcher, et al., 1998; Rugg, Fletcher, Chua, \& Dolan, 1999).

\section{DISCUSSION}

The present study provides evidence in support of our hypothesis that activation of the right dorsolateral prefrontal cortex during episodic retrieval reflects the degree of retrieval monitoring (Henson et al., 1999a; Henson et al., 1999b; Fletcher, Shallice, Frith, Frackowiak, \& Dolan, 1998). This region showed a greater response for correct low- than correct high-confidence judgements. As noted in the Introduction, low-confidence judgments are situations where, according to a signal detection model of recognition, memory strength is close to the old-new response criterion. According to our hypothesis, these situations are likely to entail more monitoring of the retrieved information before a decision is made, consistent with the longer reaction times we observed for low-confidence judgements. Alternative interpretations of retrieval-related right prefrontal activations, such as retrieval mode (Kapur et al., 1995), or retrieval success (Rugg et al., 1996), would appear unable to explain this finding.

\section{Retrieval Monitoring}

Other interpretations of our right dorsolateral confidence effect are worth considering, however. One might argue that the more people engage in retrieval monitoring, the more information they retrieve, or the more accurate their final decision, and these situations should result in high- (not low-) confidence judgments. However, more monitoring does not necessarily entail more information being retrieved or more accurate decisions. Monitoring might result in further retrieval attempts, but these may or may not be successful. Rather, the present hypothesis is that the degree of monitoring depends on how much information is retrieved relative to the response criterion, which is a function of both the specific item and the task instructions (e.g., the relative priority of hits versus false alarms; see also Wagner, Desmond, Glover, \& Gabrieli, 1998). Furthermore, retrieval monitoring will presumably take time, which is consistent with findings that high-confidence judgments are typically made faster (not slower) than low-confidence judgments.

Another interpretation of our right dorsolateral confidence effect is that it reflects greater retrieval "effort" (Schacter, Curran, Galluccio, Milberg, \& Bates, 1996). We are not aware of any theoretical attempts to define "retrieval effort" (at least to the same extent that "retrieval monitoring" is defined within the model of Burgess \& Shallice, 1996). One might take reaction times 
as an operational definition of "effort", in which case it becomes difficult to unconfound retrieval effort and retrieval monitoring in the present experiment, or in most normal situations (given that more monitoring tends to imply longer reaction times). However, a failure of monitoring would seem better suited to explain the memory deficits following frontal lesions (even though the precise site and extent of these lesions is often unclear), which include increased susceptibility to interference (Shimamura, Jurica, Mangels, Gershberg, \& Knight, 1995), excessive repetitions during recall (Stuss et al., 1994) and high rates of false alarms (Schacter, Curran, et al., 1996).

We have argued that monitoring is engaged whenever the results of an initial retrieval attempt are ambiguous (i.e., close to the response criterion). At least three different types of process can follow. One might just use the information already obtained in an attempt to judge more precisely its relation to the response criterion. Alternatively, one might use meta-memory strategies to inform a decision (e.g., "if I had seen that word before, I would have surely remembered it"). Finally, one might make further retrieval attempts, perhaps utilizing different cues, in iterative process of memory reconstruction (Norman \& Bobrow, 1979). In this case, the extent of retrieval monitoring will correlate with the extent of retrieval cueing (or "memory search"). While we cannot fully dissociate these two processes on the basis of the present results, others have argued from imaging studies of recall tasks that a more ventral region of the posterior right prefrontal cortex (BA 45/47) is involved in retrieval cueing (Fletcher et al., 1998). Moreover, the extent of different retrieval cues for simple yes/ no recognition tasks is unclear (by relying predominantly on "copy cues" of the stimuli, recognition tasks, unlike recall tasks, would not appear to entail substantial trial-by-trial variation in cueing strategies).

Finally, we note that a homologous region in the left dorsolateral prefrontal cortex also showed a greater response to low- versus high-confidence judgments. This region did not show a significantly greater response to $\mathrm{K}$ than $\mathrm{R}$ judgments in our previous study (Henson et al., 1999a), but was activated during a previous manipulation of monitoring (Henson et al., 1999b). The present findings suggest that there are conditions under which monitoring of retrieval engages bilateral dorsolateral prefrontal regions, contrary to the right-lateralization usually associated with imaging studies of episodic retrieval (according to the "HERA" generalization, Tulving, Kapur, Craik, Moscovitch, \& Houle, 1994).

\section{Retrieval Success}

The present study provides a convincing replication of the main results from our previous study, which used Remember/Know judgements rather than confidence judgements (Henson et al., 1999a). The left-lateralized network of the posterior cingulate, precuneus, lateral parietal, and anterior prefrontal regions revealed by the present old versus new comparison was also identified in our previous study, where it was revealed in the comparison of both $\mathrm{R}$ versus $\mathrm{N}$ and $\mathrm{R}$ versus $\mathrm{K}$ judgments. The latter finding is consistent with the fact that the majority of old judgements in the present study were made with high confidence, and were therefore likely to be based on explicit recollection of the word's prior occurrence. While we cannot fully distinguish an explanation in terms of recollection (Yonelinas et al., 1996) from one in terms of high memory strengths or familiarity levels (Donaldson, 1996), we note that many of the above regions have also been associated with recollection in previous studies. The left anterior prefrontal cortex, for example, has been associated with source retrieval (Rugg et al., 1999), and so-called "reflective" retrieval processes (Nolde, Johnson, \& D'Esposito, 1998; Nolde, Johnson, \& Raye, 1998). The precuneus has been associated with retrieval of visual imagery (Fletcher, Shallice, Frith, Frackowiak, \& Dolan, 1996; though see Buckner, Raichle, Miezin, \& Petersen, 1996), which is likely to be a component of recollection, and left lateral parietal activations may underlie the left temporo-parietal ERP difference observed between correct old and new judgements (Rugg, 1995), which has also been attributed to recollection (e.g., Wilding, Doyle, \& Rugg, 1995; Smith, 1993). The posterior cingulate activation might reflect retrieval of contextual (source) information from the medial temporal cortex, which is again likely to form the basis of recollection, and is consistent with reports that lesions to the nearby retrosplenial areas result in amnesia (Rudge \& Warrington, 1993; Valenstein, Bowers, Varfaellie, Day, \& Watson, 1987).

In addition to the above findings, we obtained preliminary evidence to suggest that a more anterior and ventral region of the right prefrontal cortex exhibits a differential response to correct old and correct new judgements. This old-new difference, like that observed in the left anterior prefrontal region, only emerged with the late response function. This finding is consistent with the generally delayed BOLD response in the right anterior prefrontal cortex observed by Schacter et al. (1997) and Buckner, Koutstaal, Schacter, Dale, et al. (1998). However, the delayed response observed by these authors was found for both old and new words, relative to fixation; they did not find any significant oldnew differences (which may reflect the relatively long SOA employed in these studies, which would reduce sensitivity to differences between event-types, Josephs \& Henson, 1999). Moreover, the interpretation of the present differential response is not straightforward, in that differential loadings on the orthogonalized late response function could reflect a difference in the magnitude as well as, or instead of, differences in the latency of responses to old and new words (see Meth- 
ods). Furthermore, while a real latency difference in BOLD response could reflect relatively delayed or prolonged neural activity in anterior prefrontal regions, the delay of approximately $3 \mathrm{sec}$ seems more likely to reflect properties of the vasculature in the anterior prefrontal regions.

The association of right anterior prefrontal cortex activation with retrieval success is consistent with many previous blocked studies using recognition memory tasks (Buckner, Koutstaal, Schacter, Wagner, \& Rosen, 1998; Rugg et al., 1996, 1997, 1999; Rugg, Fletcher, et al., 1998; though see Kapur et al., 1995; Nyberg et al., 1995). Studies using word-stem cued recall, however (Allan, Dolan, Fletcher, \& Rugg, 2000; Rugg, Fletcher, et al., 1998; Rugg, Schloerscheidt, \& Mark, 1998), have not revealed the same correlation of right anterior prefrontal activation with retrieval success. In these studies, the anterior region was more active during blocks in which none of the stems could be completed with old words. Assuming that subjects employ a generate-and-recognize strategy for word-stem cued recall, more completions are likely to be generated on average for stems with completions that cannot be recognized. This suggests that the anterior prefrontal cortex may reflect the amount of information retrieved (from either semantic or episodic memory), regardless of whether that information is sufficient for successful performance of an episodic retrieval task.

Relatively delayed and prolonged differential ERPs between correct old and new judgements have been observed at frontal electrodes (the difference emerging approximately $500 \mathrm{msec}$ poststimulus and lasting for at least $2 \mathrm{sec}$ ), and are most notable over the right hemisphere (Wilding \& Rugg, 1996). These ERP effects have been attributed to cognitive operations that follow recollection (Rugg, Schloerscheidt, et al., 1998). However, the neural generators of the ERP old-new effect have yet to be localized precisely, and right frontal ERP effects could reflect activity in one or both of the right prefrontal regions (dorsolateral/ anterior) identified in the present study. Indeed, a recent ERP study (Rugg, Allan, \& Birch, 2000) found a greater right frontal old-new effect associated with shallowly, rather than deeply, studied words, which may reflect less confident recognition decisions and possibly a generator in the same right dorsolateral prefrontal region implicated by the present study. Thus, the relationship between fMRI and ERP findings will be difficult to determine if the right frontal ERP effect observed during episodic retrieval reflects multiple, functionally distinct right prefrontal generators.

The orthogonal comparisons of the main effects of low- versus high-confidence and old versus new judgments afforded by the present design represent a considerable improvement over our previous recognition studies, in which retrieval monitoring was either con- founded with retrieval success (at least for source information; Henson et al., 1999b), or inversely related to recollection (Henson et al., 1999a). One caveat concerning the present results, however, is that the unbalanced response frequencies meant that we were unable to examine whether the main effects of lowversus high-confidence and old versus new judgments were qualified by a significant interaction (though for the right dorsolateral region at least, our data suggest that a confidence effect is found even for new words alone). Thus, a more powerful future study may reveal that one or more of the regions identified in the present study show enhanced responses specific, for example, to low-confidence old judgments. Nonetheless, the present results represent the best evidence to date for a dissociation between retrieval monitoring and retrieval success.

\section{Conclusion}

We suggested previously (Henson et al., 1999b) that at least three functionally dissociable right prefrontal subregions can be identified during episodic retrieval: dorsolateral (BA 9/46), ventrolateral (BA 45/47), and anterior (BA 10). The present data support a dissociation between dorsolateral and anterior regions. The retrieval monitoring hypothesis, as presently articulated, can account for responses in dorsolateral regions. A retrieval success hypothesis seems better suited to explain the responsiveness of anterior regions, consistent with its activation in previous blocked recognition memory studies. These results converge with those from other studies to indicate that the right prefrontal cortex should not be regarded as equipotential in its role during episodic memory retrieval: Different subregions of the prefrontal cortex appear to subserve different aspects of the retrieval process.

\section{METHODS \\ Participants}

Twelve right-handed participants (nine male), aged 2132 (with a mean of 27), gave informed consent to participate in the experiment. All reported themselves to be in good health and with no history of neurological illness.

\section{Procedure}

Participants were scanned during two sessions, the Study and Test conditions, which were separated by approximately $60 \mathrm{sec}$ of backward counting. One hundred eighty 5-letter nouns with a Kucera-Francis written frequency of 10-100 were obtained from the MRC psycholinguistics database (http://www.psy.uwa.edu.au/ uwa_mrc.htm), 90 of which were assigned randomly to 
the study list for each participant (the "old" words). Old words were randomly intermixed with the remaining 90 "new" words for the test list. The stimuli were presented in 24-point Helvetica font on a Macintosh computer, projected onto a mirror approximately $300 \mathrm{~mm}$ above the participant in the MRI scanner. The horizontal visual angle subtended by the stimuli was approximately $2^{\circ}$. The words were presented for $1 \mathrm{sec}$, followed by a central fixation cross for a random interval between 2.5 and $3.5 \mathrm{sec}$ (mean SOA of $4 \mathrm{sec}$ ). A short SOA was chosen in order to maximize sensitivity to differences in the BOLD response to different event-types (Josephs \& Henson, 1999), with a concomitant reduced sensitivity to the common (main) effect.

In the Study condition, participants pressed a key with either the index or middle finger of their right hand to indicate whether the word was "pleasant" or "unpleasant"; a task that ensures reasonably elaborate semantic encoding of stimuli (Warrington \& Ackroyd, 1975; results from the Study condition are not reported here). In the Test condition, participants pressed a key with one of the four fingers of their right hand depending on whether they recognized a word from the previous Study condition and whether their judgement was made with high or low confidence. The four response keys were arranged either left-to-right or right-to-left as HighOld, Low-Old, Low-New, and High-New judgements. The direction of finger assignment, and that of the pleasant/ unpleasant judgement during study, were counterbalanced across participants.

\section{fMRI Scanning Technique}

A 2T Siemens VISION system (Siemens, Erlangen, Germany) was used to acquire both T1 anatomical volume images $(1 \times 1 \times 1.5 \mathrm{~mm}$ voxels $)$ and $\mathrm{T} 2 *$-weighted echoplanar (EPI) images $\left(64 \times 643 \times 3 \mathrm{~mm}^{2}\right.$ pixels, $\mathrm{TE}=40 \mathrm{msec}$ ) with blood oxygenation level dependent (BOLD) contrast. Each echoplanar image comprised 30 $2.4-\mathrm{mm}$ axial slices taken every $3.6 \mathrm{~mm}$, positioned to cover the cortex (the cerebellum was not imaged). Data were acquired during two sessions, consisting of 125 volumes during Study and 305 volumes during Test, of which the first five volumes per session were discarded to allow for T1 equilibration effects. Volumes were acquired continuously with an effective repetition time (TR) of $3 \mathrm{sec} /$ volume. A random jitter of $-0.5,0$, or 0.5 sec in the SOA allowed an effective sampling rate of the hemodynamic response of $2 \mathrm{~Hz}$.

\section{Preprocessing}

To correct for their different acquisition times, the signal measured in each slice was shifted relative to the acquisition of the middle slice using a sinc interpolation in time. All volumes were then realigned to the first volume and resliced using a sinc interpolation in space.
Each volume was normalized to a standard EPI template volume (based on the MNI reference brain, Cocosco, Kollokian, Kwan, \& Evans, 1997) of $3 \times 3 \times 3 \mathrm{~mm}^{3}$ voxels in the space of Talairach and Tournoux (1988) using nonlinear basis functions. The T1 structural volume was coregistered with the mean realigned EPI volume and normalized with the same deformation parameters. Finally, the EPI volumes were smoothed with an 8-mm FWHM isotropic Gaussian kernel to accommodate further anatomical differences across participants, and proportionally scaled to a global mean of 100. Though the effects of global normalization need to be interpreted with care when the global estimate is significantly correlated with the covariates of interest (Aguirre, Zarahn, \& D'Esposito, 1998), in the present case, the absolute correlations between each of the covariates of interest and the global estimate were small (mean across covariates and participants $=0.06$, maximum $=0.27$, and, more importantly, there were no reliable global estimate correlations with the four planned comparisons of covariates across participants (i.e., differential correlations between the events of interest), $t(12)<1.48, p>.10$.

\section{Data Analysis}

Data were analyzed using Statistical Parametric Mapping (SPM99, Wellcome Department of Cognitive Neurology, London, UK; Friston et al., 1995). Population inferences were made through a two-stage procedure. In the first-stage models, the volumes acquired during each session were treated as a time series, and the BOLD response to the stimulus onset for each eventtype was modeled with two response functions. These functions were a canonical hemodynamic response function (HRF; Friston et al., 1998), and a delayed HRF, shifted 3 sec later in time (i.e., by one TR). Note that these response functions make assumptions about the shape of the BOLD response, and will not capture responses in brain regions that show different forms. The functions were convolved with the event-train of stimulus onsets to create covariates in a general linear model, together with a constant term for each participant. Given that the early and late HRFs were correlated, covariates for the late HRF were orthogonalized with respect to those for the early HRF using a GramSchmidt procedure (loadings on the early covariate thus represent variance that is not shared with the orthogonalized late covariate, Andrade, Parades, Roulette, \& Poline, 1999). The data were highness filtered to a maximum of $1 / 150 \mathrm{~Hz}$. Parameter estimates for each covariate were calculated from the least mean squares fit of the model to the time series. Linear combinations of these parameter estimates represented summary statistics that comprised the data for the second-stage of repeated-measures analyses (Frison \& Pocock, 1992). 
Covariates of interest were restricted to correct old or new judgments. The small and variable number of correct low-confidence old judgements across participants (see Results), precluded a full factorial analysis (the differing efficiencies of parameter estimation across participants potentially violating the assumption of identically distributed error variance in the secondstage tests). To test the main effects of confidence or retrieval success, separate first-stage models were constructed in which judgements were collapsed across old and new words, or across high- and low-confidence judgements. To test the simple effect of confidence on new words alone (the proportions of which were approximately balanced across participants), a third model was constructed in which highand low-confidence correct new judgments were modeled separately.

These planned contrasts were performed for both early and (orthogonalized) late response functions. Note that, while tests on the orthogonalized late covariate are independent of those performed on the early covariate, the interpretation of a differential loading of two event-types on the orthogonalized late covariate could reflect differences in the timing as well as, or instead of, differences in the magnitude of the two responses. The corresponding linear combination of parameter estimates for each contrast were stored as separate images for each participant. These contrast images were entered into one-sample $t$ tests (in which participants are treated as a random variable), subsequently transformed into statistical parametric maps (SPMs) of the $Z$ statistic. The significance of these SPMs was assessed by "masking" them with those obtained from analogous contrasts in our previous study (see Results). This masking procedure restricts voxels in the "planned" contrast to those that survive a specified threshold (uncorrected $p<.01$ ) in the "mask" contrast. This procedure provides a hypothesis-driven approach to minimizing type I error, in which $p$ values for the planned contrast can be corrected for the volume of the regions of interest defined by the mask (a function of the shape, smoothness, and number of voxels in the region, Worsley et al., 1995). A list of regions that survived $p<.001$ uncorrected, but did not survive the masking or smallvolume corrections, is available from the authors on request. The maxima of suprathreshold regions were localized on the normalized structural images and labeled using the best approximation to the systems of Talairach and Tournoux (1988) and Brodmann (1909), for consistency with previous studies. The best-fitting differential event-related responses for these regions (shown in Figures 2-4) were reconstructed by summation of the early and orthogonalized late response functions weighted by the mean difference in early and late parameter estimates across participants.

\section{Acknowledgments}

This work was supported by Wellcome Trust Grant 051028/Z. RJD and MDR are supported by the Wellcome Trust. We would like to thank J.-B. Poline and A. Holmes for statistical advice.

Reprint requests should be sent to: Dr. R. Henson, Wellcome Department of Cognitive Neurology, 12 Queen Square, London, WC1N 3BG, UK, or r.henson@ucl.ac.uk.

\section{REFERENCES}

Aguirre, G. K., Zarahn, E., \& D’Esposito, M. (1998). The inferential impact of global signal covariates in functional neuroimaging studies. Neuroimage, 8, 302-306.

Allan, K., Dolan, R. J., Fletcher, P. C., \& Rugg, M. D. (2000). The role of anterior right prefrontal cortex in episodic retrieval. Neuroimage, 11, 217-227.

Andrade, A., Parades, A.-L., Roulette, S., \& Poline, J.-B. (1999). Ambiguous results in functional neuroimaging data analysis due to covariate correlation. Neuroimage, 10, 483-486.

Atkinson, R. C., \& Juola, J. F. (1974). Search and decision processes in recognition memory. In D. H. Krantz, R. C. Atkinson, R. D. Luce, \& P. Suppes (Eds.), Contemporary developments in mathematical psychology: Vol. 1. Learning, memory and thinking. San Francisco: Freeman.

Brodmann, K. (1909). Vergleichende Lokalisationslebre der Grosshirnrinde in ibren Prinzipien dargelstellt auf Grund des Zellesbaues. Leipzig: Barth.

Buckner, R. L., \& Koutstaal, W. (1998). Functional neuroimaging studies of encoding, priming, and explicit memory retrieval. Proceedings of the National Academy of Sciences, U.S.A., 95, 891-898.

Buckner, R. L., Koutstaal, W., Schacter, D. L., Dale, A. M., Rotte, M., \& Rosen, B. R. (1998). Functional-anatomic study of episodic retrieval: II. Selective averaging of event-related fMRI trials to test the retrieval success hypothesis. Neuroimage, 7, 163-175.

Buckner, R. L., Koutstaal, W., Schacter, D. L., Wagner, A. D., \& Rosen, B. R. (1998). Functional-anatomic study of episodic retrieval: I. Retrieval effort versus retrieval success. Neuroimage, 7, 151-162.

Buckner, R. L., Raichle, M. E., Miezin, F. M., \& Petersen, S. E. (1996). Functional imaging studies of memory retrieval for auditory words and visual pictures. Journal of Neuroscience, 16, 6219-6235.

Burgess, P. W., \& Shallice, T. (1996). Confabulation and the control of recollection. Memory, 4, 359-411.

Cocosco, C. A., Kollokian, V., Kwan, R. K. S., \& Evans, A. C. (1997). Brainweb: Online interface to a 3D MRI simulated brain database. Neuroimage, 5, 425.

Dale, A. M., \& Buckner, R. L. (1997). Selective averaging of rapidly presented individual trials using fMRI. Human Brain Mapping, 5, 329-340.

Donaldson, W. (1996). The role of decision processes in remembering and knowing. Memory and Cognition, 24, $523-533$

Fletcher, P. C., Frith, C. D., \& Rugg, M. D. (1997). The functional neuroanatomy of episodic memory. Trends in Neuroscience, 20, 213-218.

Fletcher, P. C., Shallice, T., Frith, C. D., Frackowiak, R. S. J., \& Dolan, R. J. (1996). Brain activity during memory retrieval: The influence of imagery and semantic cueing. Brain, 119, 1587-1596.

Fletcher, P. C., Shallice, T., Frith, C. D., Frackowiak, R. S. J., \& Dolan, R. J. (1998). The functional roles of the prefrontal cortex in episodic memory: II. Retrieval. Brain, 121, 1249-1256. 
Frison, L., \& Pocock, S. J. (1992). Repeated measures in clinical trials: Analysis using mean summary statistics and its implications for design. Statistics in Medicine, 11, 1685-1704.

Friston, K. J., Fletcher, P., Josephs, O., Holmes, A., Rugg, M. D., \& Turner, R. (1998). Event-related fMRI: Characterizing differential responses. Neuroimage, 7, 30-40.

Friston, K. J., Holmes, A. P., Worsley, K. J., Poline, J. B., Frith, C. D., \& Frackowiak, R. S. J. (1995). Statistical parametric maps in functional imaging; a general linear approach. Human Brain Mapping, 2, 189-210.

Green, D. M., \& Swets, J. A. (1966). Signal detection theory and psychophysics. New York: Wiley.

Henson, R. N. A., Rugg, M. D., Shallice, T., Josephs, O., \& Dolan, R. J. (1999a). Recollection and familiarity in recognition memory: An event-related fMRI study. Journal of Neuroscience, 19, 3962-3972.

Henson, R. N. A., Shallice, T., \& Dolan, R. J. (1999b). Right prefrontal cortex and episodic memory retrieval: A functional MRI test of the monitoring hypothesis. Brain, 122, $1367-1381$.

Jacoby, L. L. (1996). Dissociating automatic and consciously controlled effects of study/test compatibility. Journal of Memory and Language, 35, 32-52.

Josephs, O., Turner, R., \& Friston, K. (1997). Event-related fMRI. Human Brain Mapping, 5, 243-248.

Josephs, O., \& Henson, R. N. A. (1999). Event-related fMRI: Modelling, inference and optimisation. Philosophical Transactions of the Royal Society of London, 354, 1215-1228

Kapur, S., Craik, F., Brown, G. M., Houle, S., \& Tulving, E. (1995). Functional role of the prefrontal cortex in memory retrieval: A PET study. NeuroReport, 6, 1880-1884.

Koriat, A., \& Goldsmith, M. (1996). Monitoring and control processes in the strategic regulation of memory accuracy. Psychological Review, 103, 490-517.

Mandler, G. (1980). Recognizing: The judgment of previous occurrence. Psychological Review, 87, 252-271.

Murdock, B. B. (1965). Signal detection theory and shortterm memory. Journal of Experimental Psychology, 70, 609-666.

Nolde, S. F., Johnson, M. K., \& D'Esposito, M. (1998). Left prefrontal activation during episodic memory: An event-related study. NeuroReport, 8, 1317-1320.

Nolde, S. F., Johnson, M. K., \& Raye, C. L. (1998). The role of prefrontal cortex during tests of episodic memory. Trends in Cognitive Sciences, 2, 399-406.

Norman, D. A., \& Bobrow, D. G. (1979). Descriptions: An intermediate stage in memory retrieval. Cognitive Psychology, $11,107-123$.

Nyberg, L., Tulving, E., Habib, R., Nilsson, L. G., Kapur, S., Cabeza, R., \& McIntosh, A. R. (1995). Functional brain maps of retrieval mode and recovery of episodic information. NeuroReport, 7, 249-252.

Rudge, P., \& Warrington, E. (1993). Selective impairment of memory and visual perception in splenial tumours. Brain, 114, 349-360.

Rugg, M. D. (1995). ERP studies of memory. In M. D. Rugg \& M. G. H. Coles (Eds.), Electrophysiology of mind (pp. 132170). Oxford: Oxford University Press.

Rugg, M. D., Allan, K., \& Birch, C. S. (2000). Electrophysiological evidence for the modulation of retrieval orientation by depth of study processing. Journal of Cognitive Neuroscience, 12, 664-678.

Rugg, M. D., Fletcher, P. C., Allan, K., Frith, C. D., Frackowiak, R. S. J., \& Dolan, R. J. (1998). Neural correlates of memory retrieval during recognition memory and cued recall. Neuroimage, 8, 262-273.
Rugg, M. D., Fletcher, P. C., Chua, P. M.-L., \& Dolan, R. J. (1999). The role of the prefrontal cortex in recognition memory and memory for source: An fMRI study. Neuroimage, 10, 520-529.

Rugg, M. D., Fletcher, P. C., Frith, C. D., Frackowiak, R. S. J., \& Dolan, R. J. (1996). Differential activation of the prefrontal cortex in successful and unsuccessful memory retrieval. Brain, 119, 2073-2083.

Rugg, M. D., Fletcher, P. C., Frith, C. D., Frackowiak, R. S. J., \& Dolan, R. J. (1997). Brain regions supporting intentional and incidental memory: A PET study. NeuroReport, 8, 1283-1287.

Rugg, M. D., Schloerscheidt, A. M., \& Mark, R. E. (1998). An electrophysiological comparison of two indices of recollection. Journal of Memory and Language, 39, 47-69.

Schacter, D. L., Alpert, N. M., Savage, C. R., Rauch, S. L., \& Albert, M. S. (1996). Conscious recollection and the human hippocampal formation: Evidence from positron emission tomography. Proceedings of the National Academy of Sciences, U.S.A., 93, 321-325.

Schacter, D. L., Buckner, R. L., Koutstaal, W., Dale, A. M., \& Rosen, B. R. (1997). Late onset of anterior prefrontal activity during true and false recognition: An event-related fMRI study. Neuroimage, 6, 259-269.

Schacter D. L., Curran T., Galluccio L., Milberg, W. P., \& Bates, J. F. (1996). False recognition and the right frontal lobe: A case study. Neuropsychologia, 34, 793-808.

Shallice, T., Fletcher, P., Frith, C. D., Grasby, P., Frackowiak, R. S. J., \& Dolan, R. J. (1994). Brain regions associated with acquisition and retrieval of verbal episodic memory. Nature, 368, 633-635.

Shimamura, A. P., Jurica, P. J., Mangels, J. A., Gershberg, F. B., \& Knight, R. T. (1995). Susceptibility to memory interference effects following frontal lobe damage: Findings from tests of paired-associate learning. Journal of Cognitive Neuroscience, 7, 144-152.

Smith, M. E. (1993). Neurophysiological manifestations of recollective experience during recognition memory judgements. Journal of Cognitive Neuroscience, 5, 1-13.

Stuss, D. T., Alexander, M. P., Palumbo, C. L., Buckle, L., Sayer, L., \& Pogue, J. (1994). Organisational strategies of patients with unilateral or bilateral frontal lobe injury in word list learning tasks. Neuropsychology, 8, 355-373.

Talairach, J., \& Tournoux, P. (1988). Co-planar stereotaxic atlas of the human brain. Stuttgart: George Thieme Verlag.

Tulving, E. (1985). Memory and consciousness. Canadian Psychologist, 26, 1-12.

Tulving, E., Kapur, S., Craik, F. I., Moscovitch, M., \& Houle, S. (1994). Hemispheric encoding/retrieval asymmetry in episodic memory: Positron emission tomography findings. Proceedings of the National Academy of Sciences, U.S.A., 91, 1989-1991.

Valenstein, E., Bowers, D., Varfaellie, M., Day, A., \& Watson, R. T. (1987). Retrosplenial amnesia. Brain, 110, 1631-1646.

Wagner, A. D., Desmond, J. E., Glover, G. H., \& Gabrieli, J. D. E. (1998). Prefrontal cortex and recognition memory: Functional-MRI evidence for context-dependent retrieval processes. Brain, 121, 1985-2002.

Warrington, E. K., \& Ackroyd, C. (1975). The effect of orienting tasks on recognition memory. Memory and Cognition, 3, 140-142.

Wilding, E. L., Doyle, M. C., \& Rugg, M. D. (1995). Recognition memory with and without retrieval of context: An eventrelated potential study. Neuropsychologia, 33, 743-67.

Wilding, E. L., \& Rugg, M. D. (1996). An event-related potential study of recognition memory with and without retrieval of source. Brain, 119, 889-905.

Worsley, K. J., Marrett, S., Neelin, P., Vandal, A. C., Friston, K. J., \& Evans, A. C. (1995). A unified statistical approach for de- 
termining significant signals in images of cerebral activation. Human Brain Mapping, 4, 58-73.

Yonelinas, A. P., Dobbins, I., Szymanski, M. D., Dhaliwal, H. S., \& King, L. (1996). Signal-detection, threshold, and dual-process models of recognition memory: ROCs and conscious recollection. Consciousness and Cognition, 5, 418-441.

Zarahn, E., Aguirre, G., \& D'Esposito, M. (1997). A trial-based experimental design for fMRI. Neuroimage, 6, 122-138. 